\title{
Hyperglycemia and acute kidney injury in critically ill children
}

\author{
Roberto Gordillo' \\ Tania Ahluwalia ${ }^{2}$ \\ Robert Woroniecki ${ }^{3}$ \\ 'Department of Pediatrics, Division \\ of Nephrology, ${ }^{2}$ Department of \\ Pediatrics, University of Illinois \\ College of Medicine, Peoria, IL, USA; \\ ${ }^{3}$ Division of Pediatric Nephrology and \\ Hypertension, Stony Brook Children's \\ Hospital, Stony Brook, NY, USA
}

Correspondence: Roberto Gordillo Department of Pediatrics, Division of Nephrology, University of Washington School of Medicine, Seattle's Children's Hospital, 4800 Sand Point Way NE, OC.9.820, Seattle, WA 98105, USA Email rogordill@gmail.com
This article was published in the following Dove Press journal: International Journal of Nephrology and Renovascular Disease 25 August 2016

Number of times this article has been viewed

Background: Hyperglycemia and acute kidney injury (AKI) are common in critically ill children and have been associated with higher morbidity and mortality. The incidence of AKI in children is difficult to estimate because of the lack of a standard definition for AKI. The pediatric RIFLE (Risk, Injury, Failure, Loss of kidney function, and End-stage kidney disease) criteria can be used to define AKI in children. Various biomarkers in urine and blood have been studied to detect AKI in critically ill children. However, it is not clear whether hyperglycemia is associated with AKI. Our objective was to evaluate the effect of hyperglycemia on kidney function and its effect on neutrophil gelatinase-associated lipocalin (NGAL) in children.

Methods: We studied retrospective and prospective cohorts of pediatric critically ill subjects admitted to the pediatric intensive care unit (PICU). We analyzed data from admission that included estimated glomerular filtration rate, plasma and urine NGAL, serum glucose and peak glycemia (highest glycemia during PICU admission), and length of hospital and PICU stay from two different institutions. Results: We found that the prevalence of hyperglycemia was $89 \%$ in the retrospective cohort and $86 \%$ in the prospective cohort, $P=0.99$. AKI was associated with peak glycemia, $P=0.03$. There was a statistically significant correlation between peak glycemia and hospital and PICU stays, $P=<0.001$ and $P<0.001$, respectively. Urine NGAL and plasma NGAL were not statistically different in subjects with and without hyperglycemia, $P=0.99$ and $P=0.85$, respectively. Subjects on vasopressors had lower estimated glomerular filtration rate and higher glycemia, $P=0.01$ and $P=0.04$, respectively. Conclusion: We conclude that in critically ill children, hyperglycemia is associated with AKI and longer PICU stays.

Keywords: AKI, hyperglycemia, NGAL, peak glycemia, eGFR, PICU

\section{Introduction}

Hyperglycemia is common in critically ill patients, and it has been associated with increased mortality and morbidity in adults and children. ${ }^{1-3}$ Srinivasan et $\mathrm{l}^{4}$ found that blood glucose level $>150 \mathrm{mg} / \mathrm{dL}$ was associated with a 3.5 -fold increased risk of mortality. In critically ill adults, a large prospective randomized clinical study showed that strict control of blood glucose with insulin therapy reduced the incidence of acute kidney injury (AKI). ${ }^{1}$ In children, the incidence of AKI is difficult to estimate because of the lack of a standard definition for AKI and lack of large prospective multicenter studies involving general intensive care population, making the AKI incidence prone to definition and center-specific bias. ${ }^{5}$ Over 30 definitions of AKI have been published, ${ }^{4}$ and pediatric AKI definition has been "extrapolated" from adult studies. AKI in children is associated with significant mortality, as high as $60 \% .^{3}$ Therefore, prevention of AKI is crucial, and includes hemodynamic support, maintenance of euvolemia, and avoidance of nephrotoxic medications. However, it 
is not clear whether hyperglycemia is associated with AKI, and whether it has an impact on AKI outcomes in critically ill children. A number of biomarker studies focused on early detection of AKI, ${ }^{6}$ and reference levels of these biomarkers in a healthy pediatric population have since been established. ${ }^{7}$ Neutrophil gelatinase-associated lipocalin (NGAL) is a protein that is overexpressed in the kidneys after ischemia, ${ }^{8}$ and elevated NGAL serum and urine levels have been used as an early biomarker of AKI. ${ }^{6}$ However, it is unclear if NGAL levels are affected by hyperglycemia in critically ill children with AKI.

The aim of our study was to evaluate the association of hyperglycemia with AKI in critically ill children and examine the effect of hyperglycemia on NGAL levels.

\section{Materials and methods}

Pediatric subjects admitted to the pediatric intensive care unit (PICU) were recruited in two cohorts in this study; retrospective cohort in University of South Alabama Children's \& Women's Hospital with recruitment between June 2007 and March 2009, and prospective cohort in Children's Hospital of Illinois between January 2012 and July 2013. The institutional review board of University of South Alabama Children's \& Women's Hospital, and the Children's Hospital of Illinois evaluated and approved this study. Informed consent was obtained from all parents in the prospective arm of the study - the retrospective arm was a chart review and no consent was required.

\section{Definitions}

We defined critically ill children as those who required mechanical ventilation within 36 hours of admission to the PICU. AKI was defined by the pediatric RIFLE (Risk, Injury, Failure, Loss of kidney function, and End-stage kidney disease) criteria. ${ }^{4}$ Peak glycemia was defined as the highest serum glucose level during PICU admission. Hyperglycemia was defined as serum glucose of $126 \mathrm{mg} / \mathrm{dL}$ and higher. ${ }^{4}$ Estimated glomerular filtration rate (eGFR) was calculated by Schwartz's formula. ${ }^{8}$

\section{Study criteria}

Children aged $1-18$ years admitted to PICU who were critically ill were included in the study. Children with the diagnosis of diabetes mellitus type 1 and 2, children who died within 48 hours of admission, children who had undergone a kidney transplant, children on renal replacement therapy, children with known chronic kidney disease, children with immediate postcardiac surgery, children with tracheostomy, and pregnant children were excluded from the study.

\section{Data collection}

Following laboratory data were collected upon admission to PICU: serum glucose, sodium, potassium, chloride, bicarbonate, creatinine, and blood urea nitrogen. Glomerular filtration rate was estimated (eGFR) based on bedside Schwartz equation 8 , and urine was collected using a Foley catheter. In the prospective cohort, $1.5 \mathrm{~mL}$ aliquots of plasma were centrifuged at $4^{\circ} \mathrm{C}$ for 15 minutes at $800 \mathrm{~g}$, and then stored at $-80^{\circ} \mathrm{C}$. Urine was also stored at $-80^{\circ} \mathrm{C}$ before shipping to Cincinnati Children's Hospital Medical Center to measure NGAL.

\section{Data analysis}

Except where otherwise noted, data were expressed as mean \pm standard deviation or as percentages. The software used for our analysis included SAS version 9.4 (SAS Institute Inc., Cary, NC, USA) and Microsoft Office Excel 2010 (Microsoft Corporation, Redmond, WA, USA). Statistical tests used included Mann-Whitney test for non-normally distributed variables, Fisher's exact test to examine the differences in proportions between the groups and Spearman correlation coefficients. All analyses were two sided unless specified otherwise. Statistical significance for all analyses was set at $P<0.05$.

\section{Results}

A total of 67 subjects met inclusion criteria; 37 were recruited retrospectively and 30 prospectively. All the subjects had respiratory failure due to various diagnoses: asthma, septic shock, and pneumonia. The incidence of hyperglycemia was 59/67 (88\%) and it was similar in the retrospective and prospective cohorts, 33/37 (89\%) and 26/30 (86\%), respectively, $P=0.99$. Table 1 shows all subject characteristics. Peak glycemia was significantly higher in subjects with AKI than in subjects without AKI. The length of hospital and PICU stays were longer in subjects with AKI, but the length of hospital stay did not reach a statistically significant difference. Likewise, serum NGAL and urine NGAL were higher in the AKI group, but did not reach a significant difference (Table 1).

Table I Subject demographics

\begin{tabular}{|c|c|c|c|}
\hline Demographic & Non-AKI $(n=42)$ & AKI $(n=25)$ & $P$-value \\
\hline Age (years) & $6.96 \pm 0.89$ & $6.2 \pm 1.01$ & $0.922 *$ \\
\hline Female (\%) & $47.6(\mathrm{~N}=20)$ & $48(N=12)$ & $0.57^{* *}$ \\
\hline Caucasian (\%) & $52.4(\mathrm{~N}=22)$ & $44(\mathrm{~N}=\mathrm{II})$ & $0.3 I^{* *}$ \\
\hline Hyperglycemia (\%) & $85.7(\mathrm{~N}=36)$ & $92(\mathrm{~N}=23)$ & $0.7^{* *}$ \\
\hline Peak glucose $(\mathrm{mg} / \mathrm{dL})$ & I84.73 (IQR 74) & 249.84 (IQR I07) & $0.003 *$ \\
\hline $\begin{array}{l}\text { Length of hospital stay } \\
\text { (days) }\end{array}$ & 25 (IQR I4) & 40.2 (IQR 3I) & $0.26 *$ \\
\hline $\begin{array}{l}\text { Length of PICU stay } \\
\text { (days) }\end{array}$ & I6.6 (IQR II) & 30.8 (IQR I8) & $0.03 *$ \\
\hline Urine NGAL (ng/mL) & 21.63 (IQR I5.8) & 407.9 (IQR III.6) & $0.47^{*}$ \\
\hline Plasma NGAL (ng/mL) & I I3.9 (IQR 85.8) & 291.4 (IQR 4I8.6) & $0.11 *$ \\
\hline
\end{tabular}

Note: *Mann-Whitney. **P-value tested by Fisher's exact test.

Abbreviations: AKI, acute kidney injury; IQR, interquartile range; PICU, pediatric intensive care unit; NGAL, neutrophil gelatinase-associated lipocalin. 
There were two deaths, one in the AKI group and the other in the non-AKI group. Subjects with hyperglycemia had significantly longer stays in PICU than subjects without hyperglycemia (Table 2). Subjects with hyperglycemia had also longer hospital stay, but it did not reach statistical significance. The correlation between peak glycemia and length of hospital stay and PICU stay was statistically significant, Spearman's rho $=0.49, P \leq 0.001$ and Spearman's rho $=0.53$, $P \leq 0.001$, respectively. In the prospective cohort, urine NGAL was higher in subjects with hyperglycemia but did not reach a statistically significant difference. Plasma NGAL also did not reach a significant difference between the prospective cohort subjects with and without hyperglycemia.

The subjects who required vasopressors had significantly higher peak glycemia and lower eGFR than the subjects who did not require vasopressors (Table 3 ). The correlation between peak glycemia and eGFR on admission and the correlation between peak glycemia and urine output during PICU stay were statistically significant, Spearman's rho= $-0.277, P=0.047$ and $0.26, P=0.039$, respectively.

Logistic regression was used to assess the effect of peak glycemia on AKI. Peak glycemia had a statistically significant effect on AKI, with an odds ratio of $1.12(1.03-1.2), P=0.0051$.

Table 2 Kidney function, NGAL, and length of hospital and PICU stays in subjects with hyperglycemia and normoglycemia

\begin{tabular}{|c|c|c|c|}
\hline Variable & Normoglycemia & Hyperglycemia & $P$-value* \\
\hline $\begin{array}{l}\text { eGRF on admission } \\
\left(\mathrm{mL} / \mathrm{min} / \mathrm{l} .73 \mathrm{~m}^{2}\right)\end{array}$ & $110.6 \pm 37$ & $111.2 \pm 56.9$ & 0.716 \\
\hline $\begin{array}{l}\text { Length of hospital } \\
\text { stay (days) }\end{array}$ & 14.5 & 32.9 & 0.07 \\
\hline $\begin{array}{l}\text { Length of PICU } \\
\text { stay (days) }\end{array}$ & 7.6 & 23.8 & 0.03 \\
\hline $\begin{array}{l}\text { Urine NGAL } \\
(\mathrm{ng} / \mathrm{mL})\end{array}$ & I6.5 (IQR 25.76) & $\begin{array}{l}\text { II9.1 } \\
\text { (IQR I6.3I) }\end{array}$ & 1.00 \\
\hline $\begin{array}{l}\text { Plasma NGAL } \\
(\mathrm{ng} / \mathrm{mL})\end{array}$ & I95.8 (IQR 326.I) & $\begin{array}{l}\text { I59.8 } \\
\text { (IQR 88.54) }\end{array}$ & 0.85 \\
\hline
\end{tabular}

Note: ${ }^{*} P$-value calculated by Mann-Whitney test.

Abbreviations: NGAL, neutrophil gelatinase-associated lipocalin; PICU, pediatric intensive care unit; eGFR, estimated glomerular filtration rate; IQR, interquartile range.

Table 3 Peak glycemia and kidney function in subjects on vasopressors and without vasopressors

\begin{tabular}{|c|c|c|c|}
\hline Variable & $\begin{array}{l}\text { Without } \\
\text { vasopressors } \\
\mathbf{N}=\mathbf{5 0}\end{array}$ & $\begin{array}{l}\text { With } \\
\text { vasopressors } \\
N=17\end{array}$ & $P$-value* \\
\hline $\begin{array}{l}\text { Peak glycemia } \\
(\mathrm{mg} / \mathrm{dL})\end{array}$ & $194.52 \pm 9.75$ & $251.7 \pm 26.03$ & 0.04 \\
\hline $\begin{array}{l}\text { eGFR }(\mathrm{mL} / \mathrm{min} / \\
\left.\mathrm{I} .73 \mathrm{~m}^{2}\right)\end{array}$ & I I $8.88 \pm 7.46$ & $88.69 \pm 13.76$ & 0.01 \\
\hline
\end{tabular}

Note: ${ }^{* P}$-value calculated by Mann-Whitney test.

Abbreviation: eGFR, estimated glomerular filtration rate.

\section{Discussion}

In summary, our study demonstrates that hyperglycemia is common in critically ill children and is associated with AKI. We showed that odds of having AKI increase $12 \%$ for an every increase in peak glycemia by $10 \mathrm{mg} / \mathrm{dL}$. We also showed that hyperglycemia has an impact on PICU length of stay. Other studies have reported that the incidence of hyperglycemia in critically ill children is between $14 \%$ and over $90 \%$, ${ }^{13}$ we found an incidence of $88 \%$. A better glycemic control in these children might improve outcomes. ${ }^{9}$

It has been reported that oxidative stress plays an important role in the complications of diabetes, ${ }^{11}$ and hyperglycemia causes oxidative stress in different ways. ${ }^{12}$ Vanhorebeek et $\mathrm{al}^{1}$ reported a rabbit mode of prolonged critical illness and found elevated plasma creatinine levels and severe morphological kidney damage that correlated with elevated cortical glucose levels. Renal cortical perfusion and oxygen delivery were lower in the rabbits with hyperglycemia. ${ }^{1}$ Mitochondrial respiratory chain activities were severely reduced in rabbits with hyperglycemia. ${ }^{1}$ Langouche et al $^{12}$ showed a protective effect of intensive insulin therapy on the vascular endothelium and hepatocyte mitochondria of critically ill subjects. We can speculate that a mitochondrial injury also occurs in critically ill children with hyperglycemia. The proximal tubule is sensitive to mitochondrial injury; this might be part of the AKI pathogenesis. ${ }^{1}$ In rabbits, it has been shown that hyperglycemia causes a morphological kidney damage that correlates with elevated glucose level and plasma creatinine, and this is associated with mitochondrial dysfunction. ${ }^{11}$ Mitochondrial injury plays an important role in AKI. ${ }^{11}$ In mice, it has been shown that hyperglycemia increases oxidative and nitrosative stress and accelerates renal injury. ${ }^{8}$ We can speculate that subjects with AKI and hyperglycemia may have kidney dysfunction at the mitochondrial level. This can also explain our finding of glycemia having inverse correlation with eGFR.

Our subjects with hyperglycemia stayed longer in the PICU than subjects with normoglycemia. It has been shown that hyperglycemia occurs more frequently in sicker patients, ${ }^{14}$ and this might explain the longer stays in hospital and PICU. As sicker patients may require cardiovascular support, it may explain the increased use of pressors in this population. We did find a significant correlation between peak glycemia and PICU/hospital stay and this is in agreement with a study of pediatric subjects. ${ }^{10,14}$

Subjects with higher glycemia were more likely to be on vasopressors; this is in agreement with other studies. ${ }^{4,9}$ Preissig and Rigby ${ }^{8}$ found that with increasing support measures (mechanical ventilation, vasopressors), the risk 
of hyperglycemia increases. ${ }^{8}$ As mentioned before, sicker subjects have higher glycemia and these subjects usually require vasopressor support. Our data suggest that subjects that were on vasopressors were sicker (lower eGFR) and had higher glycemia; this is in agreement with other studies. ${ }^{4,9}$

Our study found no significant difference in urine and plasma NGAL between the subjects with and without hyperglycemia, and subjects with and without AKI. It is possible that the injury caused by hyperglycemia in the mitochondria does not cause overexpression of NGAL. Another explanation could be the small number of subjects in this study.

\section{Limitations}

This study was limited by the small sample size. None of our subjects had kidney biopsy as they are rarely performed in patients with AKI. We cannot therefore, make a statement about structural/mitochondrial damage, as this is an observational study, cause-effect relationship cannot be ascertained. Another limitation is that we have no data on steroid and intravenous dextrose use in our subjects. It has been shown that hyperglycemia is more common in critically ill children ${ }^{9}$ and also with the use of steroids for various indications, such as shock and asthma, and all of our subjects were ventilated. The use of dextrose in intravenous fluids might explain the high incidence of hyperglycemia in these children. However, both of our groups were ventilated and we do not suspect that steroid/dextrose was used preferentially in one group over the other creating systematic bias.

\section{Conclusion}

Despite those limitations, to the best of our knowledge, this is the first study that describes relationship of AKI and hyperglycemia in critically ill children. We conclude that critically ill children with hyperglycemia have higher incidence of AKI and longer PICU stays. Peak glycemia correlates with lower eGFR on admission. We acknowledge that several variables might affect kidney function in these subjects; however, confirmation of these findings in larger prospective studies may provide clinical evidence for serum glucose as a biomarker of risk for AKI in critically ill children and lay foundation to clinical intervention trials to tightly regulate glycemia in selected critically ill children.

\section{Author contribution}

All authors contributed in data analysis and revised the paper.

\section{Disclosure}

The authors report no conflicts of interest in this work.

\section{References}

1. Vanhorebeek I, Gunst J, Ellger B. Hyperglycemic kidney damage in an animal model of prolonged critical illness. Kidney Int. 2009;76(5):512-520.

2. Akcan-Arikan A, Zappitelli M, Loftis LL, Washburn KK, Jefferson LS, Goldstein SL. Modified RIFLE criteria in critically ill children with acute kidney injury. Kidney Int. 2007;71(10):1028-1035.

3. Flynn JT. Choice of dialysis modality for management of pediatric acute renal failure. Pediatr Nephrol. 2002;17(1):61-69.

4. Srinivasan V, Spinella PC, Drott HR, Roth CL, Helfaer MA, Nadkarni V. Association of timing, duration, and intensity of hyperglycemia with intensive care unit mortality in critically ill children. Pediatr Crit Care Med. 2004;5:329-336.

5. Basu RK, Kaddourah A, Terrell T, et al. Prospective Pediatric AKI Research Group (ppAKI). Assessment of Worldwide Acute Kidney Injury, Renal Angina and Epidemiology in critically ill children (AWARE): study protocol for a prospective observational study. BMC Nephrol. 2015;16:24.

6. Al-Ismaili Z, Palijan A, Zappitelli M. Biomarkers of acute kidney injury in children: discovery, evaluation, and clinical application. Pediatr Nephrol. 2011;26:29-40.

7. Bennett MR, Nehus E, Haffner C, Ma Q, Devarajan P. Pediatric reference ranges for acute kidney injury biomarkers. Pediatr Nephrol. 2015; 30(4):677-685.

8. Preissig C, Rigby M. Pediatric critical illness hyperglycemia: risk factors associated with development and severity of hyperglycemia in critically ill children. J Pediatr. 2009;155(5):734-739.

9. Faustino EV, Apkon, M. Persistent hyperglycemia in critically ill children. J Pediatr. 2005;146(1):30-34.

10. Ceriello A. New insights on oxidative stress and diabetic complications may lead to a "causal" antioxidant therapy. Diabetes Care. 2003; 26:1589-1596.

11. Yoh K, Hirayama A, Ishizaki K, et al. Hyperglycemia induces oxidative and nitrosative stress and increases renal functional impairment in Nrf2-deficient mice. Genes Cells. 2008;13:1159-1170.

12. Langouche L, Vanhorebeek I, Vlasselaers D, et al. Intensive insulin therapy protects the endothelium of critically ill patients. J Clin Invest. 2005; 115(8):2277-2286.

13. Klein GW, Hojsak JM, Schmeidler J, Rapaport R. Hyperglycemia and outcome in the pediatric intensive care unit. JPediatr. 2008;153:379-384.

14. Mishra J, Dent C, Tarabishi R, et al. Neutrophil gelatinase-associated lipocalin (NGAL) as a biomarker for acute renal injury after cardiac surgery. Lancet. 2005;365(9466):1231-1238.

\section{Publish your work in this journal}

The International Journal of Nephrology and Renovascular Disease is an international, peer-reviewed open access journal focusing on the pathophysiology of the kidney and vascular supply. Epidemiology, screening, diagnosis, and treatment interventions are covered as well as basic science, biochemical and immunological studies. The manuscript

\section{Dovepress}

management system is completely online and includes a very quick and fair peer-review system, which is all easy to use. Visit http://www. dovepress.com/testimonials.php to read real quotes from published authors. 